\title{
THE SMALLEST GRAPH OF GIRTH 5 AND VALENCY 4
}

\author{
BY NEIL ROBERTSON
}

Communicated by V. Klee, June 8, 1964

In what follows a graph $S$ is constructed which has 19 vertices, valency 4 and girth 5 . It is established that, to an isomorphism, $S$ is the only graph with less than 20 vertices of valency 4 and girth 5 . Some of its elementary properties are pointed out. This graph represents a continuation of the studies by Tutte [3], McGee [1] and Singleton [2], on graphs with specified valency and girth containing a relatively small number of vertices.

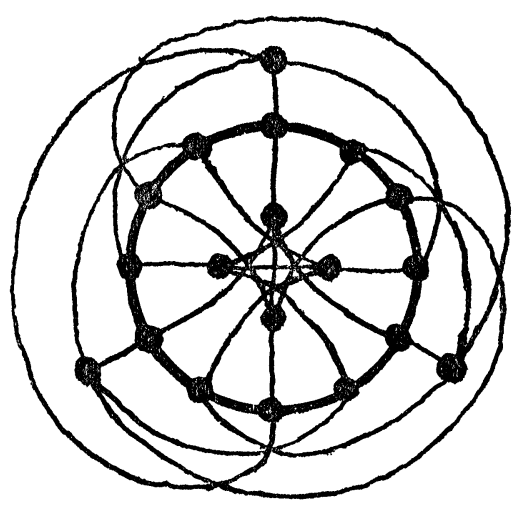

The Graph $S$

Suppose $G$ is a graph satisfying the stated constraints. The arcs of length 2 from any vertex $x$ in $G$ form a subtree $T(G, x)$ with 17 vertices. If $G$ contains only these 17 vertices, count the pentagons through an edge $A$ incident with $x$. Counting the arcs of length 2 proceeding away from the end of $A$ opposite to $x$, it is clear that there are 9 pentagons through $A$. There must then be $9 \cdot 17 \cdot \frac{4}{5} \cdot 2$ pentagons in $G$, which is absurd. When $G$ has 18 vertices each edge is contained in 8 pentagons, giving $8 \cdot 18 \cdot \frac{4}{5} \cdot 2$ pentagons in $G$, which is again impossible. $G$ must thus contain at least 19 vertices. In this event let the 2 vertices outside $T(G, x)$ be nonadjacent for each vertex $x$ in $G$. Then each edge is in 7 pentagons and $G$ contains $7 \cdot 19 \cdot \frac{4}{5} \cdot 2$ pentagons. This contradiction implies that for some $x$ in $G$ the 2 extra vertices must be adjacent. There are only two essentially different ways in which their edges can be connected to $T(G, x)$. One of these configurations completes uniquely to the graph $S$ while the other cannot be 
completed within the constraints. The arguments involved are sufficiently routine to be omitted here.

Examining all $T(S, x)$ it is seen that $S$ has three types of vertices. In the diagram they are arranged 12 on, 4 in and 3 outside the distinguished dodecagon. The automorphism group of the graph is identical with that of the dodecagon, hence it is the dihedral group of order 24. Four types of edges $A, B, C$ and $D$ are found in the graph. They are arranged 2 disjoint from, 12 incident inside, 12 on and 12 incident outside of the dodecagon. There are 9 pentagons through each of the 2 edges of type $A$ and 7 through each of the 36 others, a total of 54 pentagons in the graph. These fall into four categories under the group of the graph: 24 have edges of type $B, C$ and $D, 12$ have no edges of type $B, 6$ have no edges of type $C$ and 12 have no edges of type $D$.

The vertices of $S$ can be assigned three colours such that each edge has different coloured ends in only one way under the automorphisms. Use white, black and red without distinguishing permutations of the colours. Two of the four inner vertices must be of one colour, say white. If as many as one of the outer vertices is not white it may be coloured black. This determines two red and two white vertices on the dodecagon. Two adjacent vertices of the dodecagon are adjacent to the red vertices. They are symmetric in the colouring so they can be assigned in either order white and black. The colouring can now be directly continued, but cannot be completed. All the outer vertices must consequently be white. The dodecagon is coloured with alternate vertices red and black. This determines the red and black inner vertices.

\section{REFERENCES}

1. W. F. McGee, A minimal cubic graph of girth seven, Canad. Math. Bull. 3 (1960), 149-152.

2. R. R. Singleton, On minimal graphs of maximum even girth, Doctoral dissertation, Princeton University, Princeton, N. J., June 1962.

3. W. T. Tutte, A family of cubical graphs, Proc. Cambridge Philos. Soc. 43 (1947), 459-474.

University of Waterloo, Waterloo, Ontario, Canada 